\section{Vibration, Resonance and the Effect on Microscopes}

\author{
Douglas A. Anderson, Ph.D. \\ Schnabel Engineering Associates \\ danderson@schnabel-eng.com
}

\section{Introduction}

When we sit down at our microscopes, we assume that the environment is ideal - clean and quiet, with little to distract us from the sample. If an obvious distraction does occur, such as a bump to the instrument, or a big power surge, we look carefully and wonder, "Has the sample been damaged? Will the photograph be messed up?"

There are disturbances we may not notice, some of which are discussed in the Microscopy Listserver. One problem I have been involved in mitigating is the degradation of image quality (primarily decreased resolution) caused by vibration. We may not be aware if we have this problem, because the body is a lot less sensitive to vibration than a microscope. I will talk about the general nature of vibration, how it affects microscopy, and some thoughts on possible mitigation techniques.

\section{Vibration}

Vibration propagates as waves. A simple example is the series of waves generated by a pebble dropped in a pond. Let's say I am fishing, with a bobber on my line. The total vibration "system" then consists of the source (the dropped pebble), the propagation path (the water), and the receiver (the bobber). The pebble displaces water, which propagates away from the entry point, and moves the bobber up and down. It is convenient to separate the whole process of vibration into these three parts: Source, Path, and Receiver.

Anything that moves can be a source of vibration. The pebble, a cooling fan, foot traffic, rotating machinery, vehicles, and nearby construction activity are some potential sources. These examples fall between two limiting cases: impulsive sources and steady-state sources. The pebble closely approximates an impulsive source, while a cooling fan is a steadystate source. Repetitive impulsive sources (such as foot traffic or repeated hammer blows) can generate vibration that is similar to steady state.

In the simple example of the pebble in a pond, the path that the waves travel along is the surface of the water. The path is much more complicated when waves travel through solid materials of varying physical properties and configurations. All discontinuities serve as reflectors and refractors, sending portions of the vibration energy in different directions. Much as echoes in an auditorium reverberate and lengthen the initial sound, the wavelet generated from an impulsive source can be transformed into a longer, more complicated wave by the effect of the path.

Finally, the bobber is a very simple receiver, moving as a solid mass relative to the still water level. More complicated receivers will respond to input vibration as parts move relative to one another. It is this internal relative motion that causes problems.

Lets apply the source/path/receiver division to a more relevant example - an electron microscope in a laboratory near a railroad line. In this case, the source is the movement of the train on the tracks. The complex interaction of the rail cars, the trucks, and the track as the train passes will generate a steadystate vibration. If there are flats on the wheels (a common occurrence) a series of impulsive sources will be also be generated. The source is then a complex combination of the steady state and impulsive vibrations. The propagation path is from the tracks, through the ground, into the foundation of the building, and then through various portions of the building. Various parts of the ground, the foundation, and the building will amplify and modify the initial vibration signature significantly. Finally, the vibrations at the base of the electron microscope will be transmitted into the various parts of the microscope, causing it in turn to respond.

Unlike the bobber, which moves as a solid mass, various portions of the column of the electron microscope will move relative to one another. If the relative movement is vertical, the focussing will be degraded, as the focal distance changes. If the relative movement is horizontal, image blurring will occur as the electron beam is moved horizontally back and forth over the sample. Small relative motions are amplified in the microscope, and this is what creates such a problem for microscopists.

A simple sinusoidal wave can be described in terms of its amplitude and frequency. The amplitude can be measured in different ways: displacement, velocity, and acceleration. One can transform from one measurement to the other by integration (from velocity to displacement, for example) or differentiation (from velocity to acceleration). These measurements, then, are better described as particle displacement, particle velocity or particle acceleration. For example, the particle displacement of the bobber in the pond is how much the bobber moves up and down as the wave passes. Remember that waves leave the system it in its atrest position after they pass.

The other parameter describing the wave is frequency. Again, for the bobber, this is how many cycles per second (or Hertz) the bobber moves up and down. For a typical vibration that is more complicated than the pebble drop, many frequencies with varying amplitudes are present. Fourier analysis can determine, for any given wave, the relative amount of vibration at all frequencies present in the wave. A Fourier Series can decompose even the most complicated-looking wave into sine waves of different frequencies.

Sometimes equipment specifications are given with vibration criteria in specific frequency bands. A useful representation is the Response Spectrum, which shows how a structure with a given resonant frequency and damping will respond to an input vibration wave. Let's say an electron microscope column resonates at $10 \mathrm{~Hz}$ due to its mechanical properties and configuration. An $8 \mathrm{~Hz}$ sine wave will excite that $10 \mathrm{~Hz}$ resonance, even though it doesn't exactly match the resonant frequency. A Fourier Transform would indicate that since the wave doesn't match the resonant frequency of the microscope, there is no problem. The response spectrum would indicate that there is a potential problem.

\section{Resonance}

Imagine a small block of Styrofoam with a stiff wire, say about a foot long, stuck in the top of it. Imagine a lump of clay at the top of the wire. This device is like an inverted pendulum, and pendulums have frequencies associated with the length and stiffness. If the block of Styrofoam is moved slowly back and forth (a "wave" moving the block), this simple apparatus moves as a whole. If it is now moved at the resonant frequency, the lump of clay will move relative to the base, with a greater displacement than is imparted to the base. 


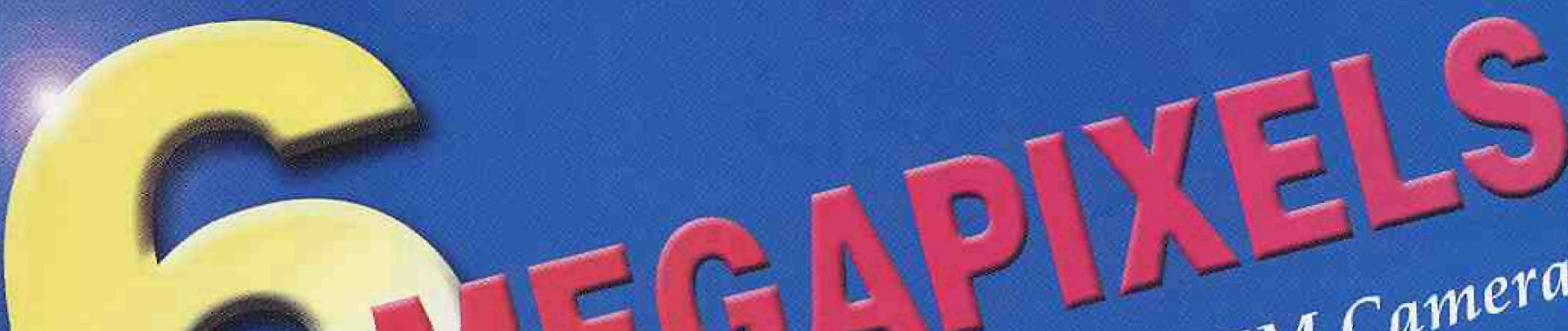

as High Defi

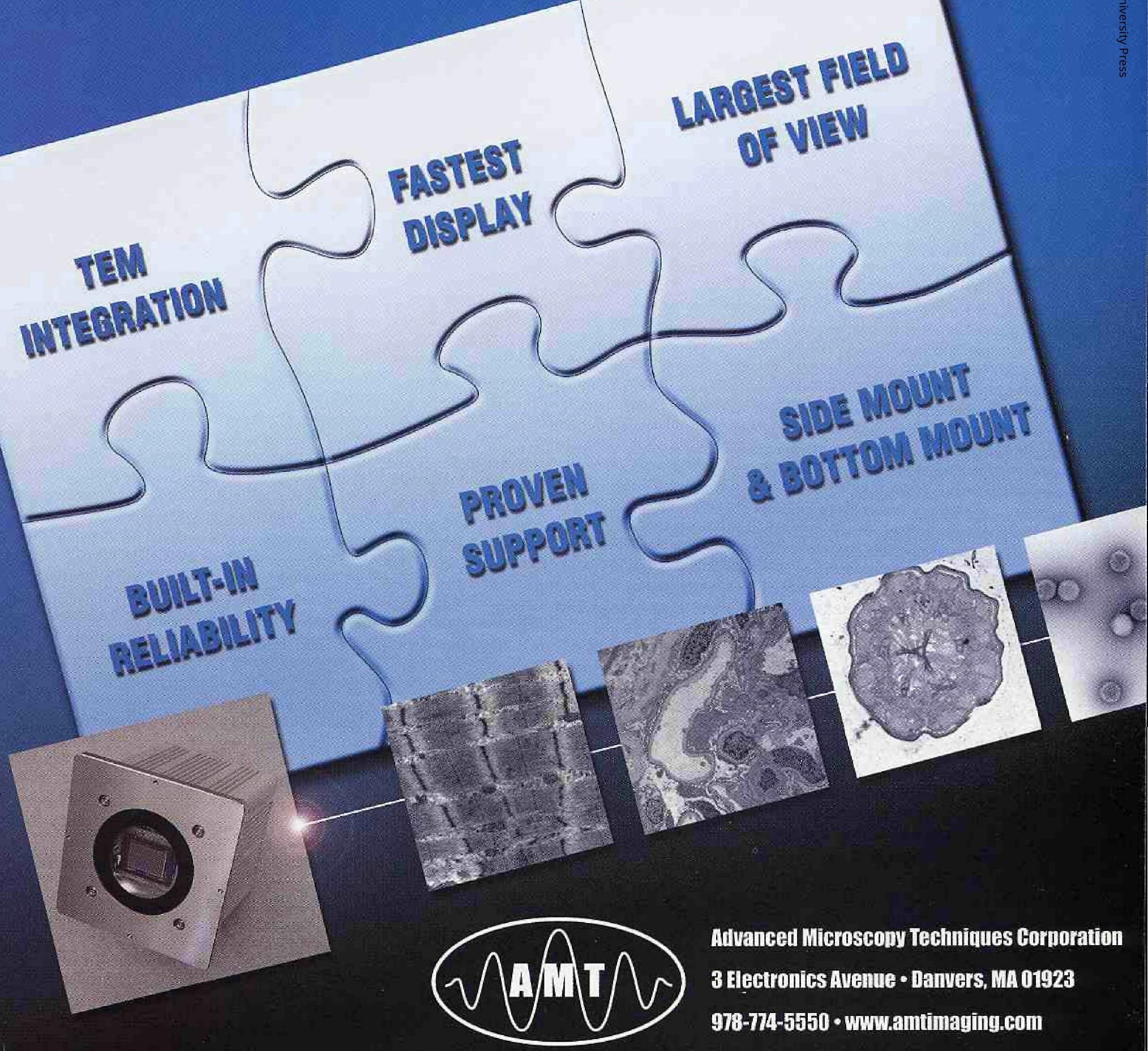
AWt's $\times 250 \& \times 13100$ 


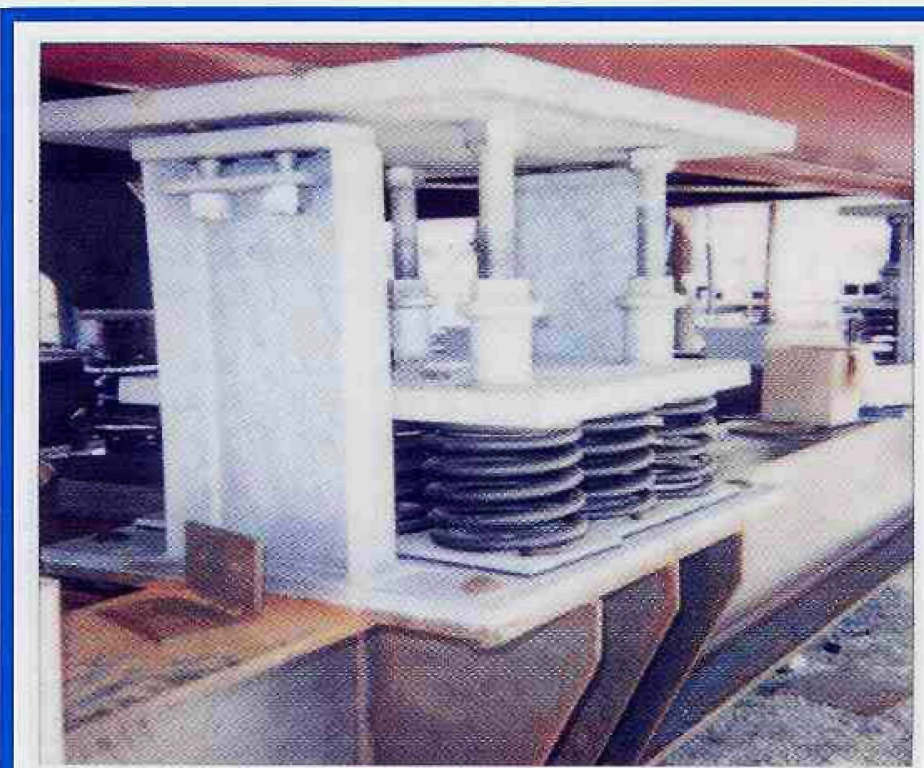

Figure 1: Anti-vibration mount.

Any freestanding structural element will respond to vibration in a manner consistent with the materials properties and configuration. A building, a bridge, a lump of clay on a stick, or an electron microscope will each have one or more resonant frequencies. In essence they are like large tuning forks - the higher the column, the lower the frequency, that it will amplify. The stiffer the material, the higher the frequency.

Of course structures that are more complicated than our inverted pendulum have more complicated responses - different "modes" of vibration. The principles remain the same. Two points are critical here: A relative motion between the base and the structure is generated, and the amount of relative motion is determined by how closely the resonant frequency of the structure matches that of the input vibration.

To determine if vibration is a problem for a particular application, measurements are needed. The key is to determine if the resonances in the system are being excited. Typically, the mechanical properties of a system are not specified, even though they are important for proper performance. The appropriate measurements are made both at the base of the system (i.e., on the floor) and on the column or support for the system. The latter would be on the column for an electron microscope, or the eyepiece of the light microscope. Again, it is relative motion in the equipment that causes image degradation.

We can compare the vibration amplitudes and frequencies at the various points in the structure and determine if there is amplification. This information is then the basis of any mitigation techniques that may be applied.

Although I am not going to try to suggest solutions for all vibration problems, I do think there are some general things to look out for, and a few things to try. Trying to reduce a vibration problem by trial-anderror can cause trouble. Simply putting a resilient material at the base to absorb vibration is not generally an appropriate solution, and in fact it can actually make the problem worse if a resonance is introduced through this material. A well-designed mitigation program can provide solutions through several means. If we break the problem down into the various parts again, an appropriate solution (or combination of solutions) can be devised.

Sometimes the source of the vibration is obvious, especially if it can be associated with time-specific events. For example, a cooling tower, elevator, nearby rail line, or the heavy plodding of a co-worker can all cause vibration problems. If, for example, a cooling tower is generating vibrations that affect several pieces of equipment, vibration mountings (Figure 1) are available to reduce the transmission of the vibration from the cooling tower to the rest of the building. These are basically simple springs, but they need to be "tuned" to the specific type of equipment (frequency, mass, etc.).

The path includes the geological path for outside sources, the foundation, and floor structures from the source to the receiver. In many cases, it is impractical to modify the path sufficiently to be able to significantly reduce vibration. Two approaches that have been successful are creating barriers from external sources and modifying floor structure.

A trench or array of caissons outside a building can be used to interrupt the path from a nearby vibration source such as a rail line. We have successfully employed such a technique (this was at an ophthalmic surgical facility), and it was significantly less expensive than special mountings for the equipment. This only works if the source is fairly close, because the caisson array can only be emplaced to a fairly shallow depth, and if the source is too far away, this array does not shadow the vibrations impacting the building.

Floors above grade level typically have resonant frequencies around $6 \mathrm{~Hz}$, resulting from the stiffness and length of the beams. Stiffeners can be added, but this is typically difficult in retrofit.

Assuming the microscope acts as a receiver, modifications are usually made to the support of the microscope. Addition of supports below the table can help for the simple case of a light microscope on a table. In this case, the trial-and-error technique can be very cost effective if time is not of the essence. The standard approach of the microscopy industry is to use a floating support. This can be quite expensive, and one needs to be sure that the system is tunable so that the specific resonances can be excluded.

A simpler version is to mount the system on springs, the same vibration mounting technique used to reduce the vibration from the source. Again, tuning the system is important, and the input source and range of frequencies of concern must be determined.

Another technique that I don't believe has been applied to the microscopy industry is dynamic vibration absorbers. These are rather simple looking devices, essentially inverted pendulums that are attached to the equipment that vibrate in response to the input and convert that vibration to heat.

\section{Conclusions}

I hope that I have given a feel for the nature of vibration generation and how microscopes can be affected. Vibration can be an unseen resolution-reducer, and it is difficult to know without actually measuring the vibration whether there is a real problem. Here are a few final suggestions:

- The equipment specifications should include the attainable resolution, and be measurable with standardization samples. If an instrument is not achieving this resolution, then vibration might be one of the contributors.

- If there is a perceptible vibration during use, be aware that the microscope might be amplifying these vibrations.

- If decreased resolution is associated with some specific event (train passing, fan running, people walking, elevator running), vibration may be the cause.

- Vibration Measurements, when made, should be at both the base level and on the microscope to determine resonances and amplification.

Be careful when attempting your own fix: what makes intuitive sense might in fact make the problem worse. 
EX CELLE N C E ... M A G N IF I E D
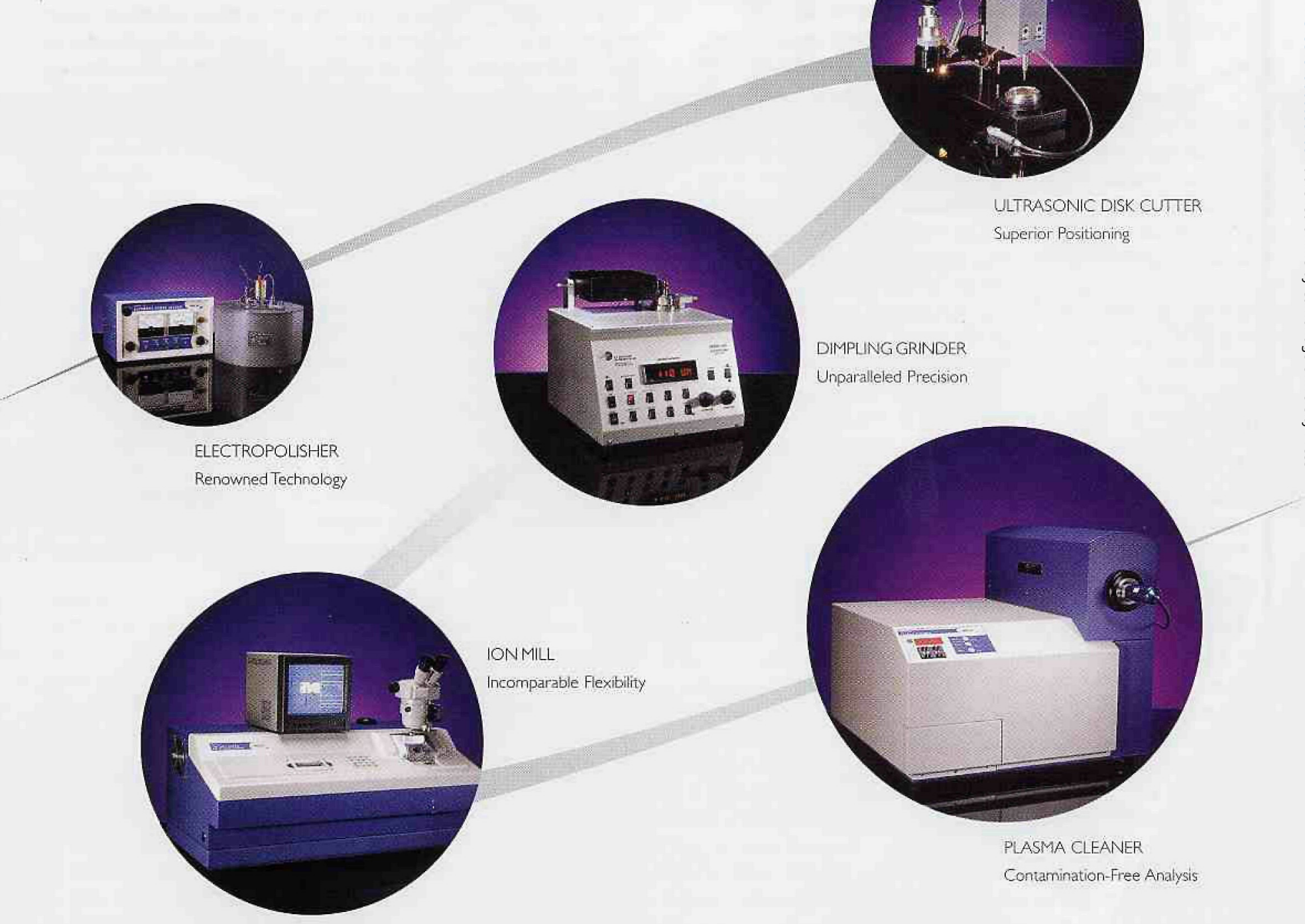

Recognized worldwide for maintaining the highest standards of quality and innovation, Fischione Instruments serves the electron microscopy community by providing state-of-theart instrumentation to meet both existing needs and the requirements of emerging microscopyrelated technologies. 